\title{
Kulturschock und Geschwisterkomplex
}

\author{
Anna Bally (Bern)
}

Zusammenfassung: Der folgende Artikel basiert auf einer kürzlich publizierten Forschungsmonografie (Bally, 2013), in welcher die Autorin in einem selbstreflexiven Forschungsprozess unter anderem der Frage nachgeht, welche Zusammenhänge sich zwischen der Geschwisterdynamik in der Herkunftsfamilie von indonesischen Migrantinnen und dem Aufbauen neuer Lateralbeziehungen in der Schweiz erkennen lassen. Das Forschungsmaterial wird in dieser Monografie aus der Perspektive unterschiedlicher Disziplinen interpretiert (Sozialanthropologie, Psychoanalyse, Geschlechterforschung) und mit der Forschung zum malaiischen Verwandtschaftssystem verknüpft. Dort lassen sich vielfache Hinweise auf die zentrale Bedeutung von Geschwisterpaaren oder-gruppen für soziale und Verwandtschaftssysteme sowie für rituelle und Alltagspraktiken finden. Anhand von ausgewählten Migrationsschicksalen wird exemplarisch eine Frage von allgemeinerer Bedeutung erforscht: diejenige nach der inneren Repräsentation der Position oder Stellung des Subjekts in der sozialen Welt. Diese innere Repräsentation ist wesentlich durch die Geschwistererfahrung und den Geschwisterkomplex strukturiert. Im vorliegenden Artikel wird ausschnittweise in diese «laterale» Perspektive eingeführt.

Schlüsselwörter: Migrationsforschung, Psychoanalyse, Sozialanthropologie, Geschwister

\section{Eine laterale Kurskorrektur}

In meinem Artikel werde ich über die Sichtweise sprechen, bei der den Geschwistern und deren «Erben» innerhalb der Familiendynamik ein eigenständiger theoretischer Status zugesprochen wird. Diese Sichtweise ist auch in der Psychoanalyse nicht grundsätzlich neu. Bereits Freud hielt Geschwister für ausserordentlich wichtig, war allerdings stärker am Ödipuskomplex interessiert. Es ist eindrücklich, wie er in einigen seiner Fallgeschichten einerseits Geschwisterfiguren oder Geschwistererfahrungen für entscheidend hielt, um andererseits diese laterale Linie im nächsten Moment gleichsam wieder zu vergessen (vgl. Freud, 1909). So wurde und wird in der psychoanalytischen Praxis selten 
konsequent und bewusst mit dieser Sichtweise gearbeitet und ebenso selten wird die «laterale» Dimension theoretisch berücksichtigt und weiterentwickelt. Dies ist zu bedauern, denn es geht beim Geschwisterkomplex um eine seelisch ähnlich entscheidende strukturelle Schwelle, wie sie der Ödipuskomplex darstellt. Beim frühen Lacan beispielsweise steht nichts Geringeres als das «sozialisierte Objekt» auf dem Spiel (Lacan, 1994, S. 61).

Bei mir ist die Ausrichtung auf die Geschwisterthematik in allen ihren seelischen und sozialen Facetten Ergebnis einer mehrjährigen ethnologischen und psychoanalytischen Forschungsarbeit mit indonesischen Frauen in der Schweiz (Bally, 2009, 2013). Durch «Anita», die Protagonistin meiner Langzeitfallstudie, wurde ich empirisch für diese so selbstverständlich für wichtig gehaltenen, wie auch rätselhaften und gleichzeitig unterschätzten «lateralen» Beziehungen (Mitchell, 2000, 2003) sensibilisiert. Ich begleitete Anita in ihrem Integrationsprozess während zweier Jahre intensiv und führte mit ihr 60 Gespräche. Die Gespräche mit Anita dienten dazu, Hypothesen zu erarbeiten, die über diesen Einzelfall hinausweisen. Meine Hypothesen habe ich anschliessend bei der Analyse von acht weiteren Fallstudien überprüft und weiter ausgearbeitet. Mit diesen acht Frauen habe ich jeweils zwei längere Gespräche im Abstand von einem Jahr geführt und ich nenne diese Fallstudien in meiner Arbeit «Migrationsbiografien».

Geschwister nahmen in den Narrativen der meisten meiner Gesprächspartnerinnen emotional viel Raum ein. Auf Geschwister wurde im Sinne von lebendiger Nähe, von Vertrautheit und Vertrauen Bezug genommen. Sie tauchten aber auch im Zusammenhang mit unüberbrückbarer Distanz, Entfremdung, Neid, Rivalität und Schuldgefühlen auf. Nicht nur Liebe, sondern auch Hass gegenüber Geschwistern wurde bei zweien der von mir befragten Frauen eindrücklich spürbar. Geschwister waren sehr präsent, sie konnten aber auch durch Abwesenheit auffallen: Nina zum Beispiel lernte ich in unserem ersten Gespräch als Einzelkind kennen, einem in Indonesien immer noch relativ seltenen Phänomen. Erst im zweiten Gespräch, ein Jahr später, erfuhr ich unverhofft von einem älteren Adoptivbruder, mit dem sie zusammen aufgewachsen war. Da ihre Mutter längere Zeit kein Kind empfangen konnte, adoptierten ihre Eltern das Baby eines Bruders der Mutter, «damit die Mutter leichter schwanger wird». Ein paar Jahre später wurde Nina geboren. Sie verbrachte also ihre gesamte Kindheit und Jugend mit ihrem älteren Adoptivbruder, dessen Existenz sie jedoch in unserem ersten langen und sehr lebhaften Gespräch verschwiegen hatte. Ein markanter Kontrast zu den Gesprächen mit Anita, in welchen ausser den Eltern auch ihre Geschwister von Anfang an benannt und mir im Sinn von Übertragungen vorgestellt wurden. 
Die entsprechende Analyse der in den Gesprächsmaterialien erkennbaren lateralen Identifizierungen, Bindungen, Fantasien usw. wurde für mich zu einer der interessantesten Aufgaben, die sich mir während meiner Forschung stellten. Im Laufe der Untersuchungen versuchte ich, Verbindungen zwischen Geschwistererfahrungen im obigen Sinne und den jeweiligen Migrationsschicksalen herauszuarbeiten. Lateralität diente mir dabei gleichsam als theoretische Klammer, mit der sich die wichtigsten Forschungsfragen und -ebenen zwanglos verbinden liessen.

Im Vergleich mit anderen Kulturen haben Geschwister in Indonesien eine sehr wichtige Funktion (vgl. Smith Kipp, 1986; Peletz, 1988; Schneider, 1993; Marschall, 2000; Feller und Zemp, 2006). Robert McKinley bezeichnet die Geschwisterbeziehungen als das soziale und verwandtschaftliche Kernkonzept malaiischer Gesellschaften (McKinley, 1983). Entlang von Genderkonstruktionen existieren in Indonesien unterschiedliche Rollenerwartungen und soziale oder ökonomische Strategien für ältere und jüngere Schwestern beziehungsweise Brüder (Feller \& Zemp, 2006; Znoj, 2009). Natürlich muss man hier beachten, dass diese Geschwisterbedeutungen auch in Indonesien einem mehr oder weniger starken Wandel unterworfen sind. Unter Berücksichtigung ethnologischer Forschungsergebnisse zur kulturellen Bedeutung von Geschwistern in Indonesien, habe ich mich bei meinen Analysen besonders dafür interessiert, welche Faktoren im Einzelfall für die Migrationsentscheidung ausschlaggebend gewesen sein könnten und mit welchen spezifischen sozialen und seelischen Herausforderungen sich die einzelnen Frauen im Verlaufe ihres Integrationsprozesses konfrontiert sahen.

Als Zuwanderinnen in den meisten Fällen anfänglich einzig und alleine auf ihre schweizerischen Ehemänner angewiesen und in einem kulturell meist ungewohnt nahen Verhältnis zu diesen lebend, müssen sich indonesische Frauen emotional und kognitiv in einer Umwelt orientieren, in der ihnen der alltägliche Rückhalt durch die Geschwisterbeziehungen fehlt.

Die Paarbeziehungen und Freundschaften der Migrantinnen habe ich als in der Nachfolge der Geschwisterbeziehungen stehend aufgefasst und die Aussagen meiner Gesprächspartnerinnen dahingehend untersucht. Weiter habe ich die Migrationserfahrung dieser Frauen als potentiell traumatisierende Wiederholung der kindlichen Entwicklungsaufgabe des Findens einer sozialen Position in der Geschwisterreihe interpretiert. Diese laterale Perspektive ermöglichte es mir, den besonderen Stellenwert von Geschwisterbeziehungen im indonesischen Kontext in Bezug auf die Migrationserfahrung zu erforschen und dadurch psychische und 
soziale Aspekte von Migrationsentscheidungen und Integrationsprozessen besser zu verstehen.

\section{$2 \quad$ Anita}

Bei meiner Probandin Anita kann die Migration als Symptom und als Wiederholung verstanden werden. Sie heiratete in Indonesien einen Schweizer und sie wusste, dass sein Aufenthalt in Indonesien zeitlich begrenzt sein würde. Anita erlebte jedoch die Folgen ihrer eigenen Entscheidung für diese Ehe und für die spätere Auswanderung in die Schweiz wie eine Einwirkung oder Manipulation von aussen: Es wurde ihr etwas zugefügt und zugemutet; es wurde ihr etwas weggenommen; sie wurde ins Exil verbannt. In diesem Exil kann sie sich nicht orientieren, sie weiss nicht mehr wer oder was sie ist und welches ihre Position ist. Die gewohnten sozialen und psychischen Strategien führen Anita weder zum erhofften beruflichen Erfolg noch zu persönlicher Zufriedenheit. Das Gefühl, nach ihrer Ankunft in der Schweiz plötzlich ein «Nichts» geworden zu sein, entstand für Anita eben nicht nur aufgrund der Tatsache, dass vieles für sie neu, fremd und schwierig war, sondern auch, weil sie gleichzeitig den Eindruck hatte, sie wäre von etwas unheimlich Vertrautem eingeholt worden: Gefühle derVernichtung hat Anita als Autobiografin in unseren Gesprächen mehrfach benannt.

Meine (Re-)Konstruktion auf der Basis des Gesprächsmaterials ergab, dass Anita durch den plötzlichen Verlust ihrer Position als anak bungsu (indonesisch: Jüngste/r einer Geschwistergruppe), damals als ihr noch jüngerer Bruder geboren wurde, überfordert war. Erschwerend war möglicherweise von Anfang an die von Anita selbst angesprochene Rolle als Ersatzkind. Anita dachte, es müssten Babybrüder gewesen sein, die vor ihrer Geburt gestorben waren und sie vermutete, dass ihre Mutter besonders deshalb von ihr als Mädchen so enttäuscht war. Zur traumatischen Katastrophe wurden diese frühen Erfahrungen aber erst nachträglich. Anita fühlte sich in ihrer geschwisterreichen Familie über mehrere Entwicklungsphasen hinweg immer wieder zurückgesetzt, verlassen, manipuliert oder ungerecht behandelt, sowohl durch Einzelne der Geschwister wie auch durch ihre Eltern. Sie konnte die Hoffnung, für ihre Mutter oder für ihren Vater im Grunde die Wichtigste und Einzige zu sein, nicht aufgeben. Anita musste an dieser Hoffnung festhalten, weil sie sich in ihrem Erleben des Geschwisterkomplexes nicht genügend aus den vernichtenden Gefühlen, neben den anderen «ein Nichts» zu sein, befreien konnte. Bei anderen, glücklicheren Kindern, entsprechen solche Gefühle eher einer vorübergehenden Krankheit, von der sie sich irgendwann erholen. 
Weil Anita, wie sich aus dem Gesprächsmaterial ableiten lässt, den Geschwisterkomplex wahrscheinlich nur ungenügend bewältigen konnte, blieb ihr als Hoffnung die Rückkehr zur Symbiose mit der Mutter. Diese war jedoch bereits wieder mit einem neuen Baby beschäftigt und Anita erlebte sich - vermutlich vor allem von diesem Moment an - als ungeliebt von der Mutter. Die Hinwendung zum «vergötterten» Vater erscheint Anita nachträglich wie eine Rettung, wenn sie über diese Phase ihrer Kindheit spricht. Er bot sich ihr sowohl als Liebesobjekt wie auch als Identifikationsfigur an. Die Identifikation mit der Mutter war gestört, die Mutter blieb für Anita das unerreichbare, verlorene Liebesobjekt, verloren zu Beginn unserer Gespräche auch im wörtlichen Sinne, da die Mutter ein Jahr zuvor gestorben war.

Erst als Anita in die Pubertät kam, wurde sie in Übereinstimmung mit kulturellen Erwartungen vor allem durch ihren nächst älteren Bruder mit der Forderung konfrontiert, sich wie eine junge Frau zu verhalten. Nun standen ihr nicht mehr alle Möglichkeiten offen. In Anitas Erleben liess ihrVater sie plötzlich fallen und so zog sie vorübergehend zu ihrer ältesten Schwester. Dort ging sie zur Schule, führte aber vor allem den Haushalt und hütete ihre Neffen. Für die letzten Schuljahre kehrte Anita noch einmal nach Hause zurück. Das Verhältnis zu ihrer Mutter verbesserte sich, Anita war damals die letzte noch zu Hause lebende Tochter, die anderen waren bereits ausgeflogen. Aber es drohten neue Konflikte: Anita hatte einen Freund, sie war im heiratsfähigen Alter und es musste erneut eine kulturell akzeptable Lösung gefunden werden. Der Vater übergab die Verantwortung für Anita nun endgültig der ältesten Schwester. Diese ermöglichte Anita eine Weiterbildung, dank der sie schliesslich eine sehr gute Arbeit fand. Für Anita bedeutete diese prestigereiche Arbeitsstelle sehr viel, nachträglich erscheint sie ihr wie ein Triumph über ihre Familie. Sie krönte diesen Triumph, indem sie sich einer durch die Schwester arrangierten Ehe widersetzte und einen Schweizer heiratete. In der für sie «besten Zeit ihres Lebens», traf sie also diese folgenreiche Wahl, um sich endgültig von den familiären Fesseln zu befreien.

Zuerst lebt sie mit ihrem Mann in Indonesien. Anita kann in dieser Zeit die Früchte ihrer Arbeit und ihrer neuen Selbständigkeit ernten, ihr Lebensstandard ist, dank ihrem Ehemann, vergleichsweise hoch. Als Frau geniesst sie mehr Freiheiten als die meisten ihrer mit indonesischen Männern verheirateten Geschlechtsgenossinnen. Mit ihren drei besten Freundinnen versteht sie sich so gut, dass der Kontakt mit ihnen auch nach Jahren in der Schweiz bestehen blieb. In vielerlei Hinsicht erlebt Anita eine unbeschwerte Zeit. Sie hat zwar wichtige innere Entwicklungsschritte nicht vollziehen können, aber aufgrund der günstigen äusse- 
ren Situation scheinen die grossen Konflikte dennoch in einem gewissen Sinne überwunden. Anita schilderte aber auch aus dieser Zeit Erfahrungen, die auf ihre grundlegende Unsicherheit und Verletzlichkeit, verborgen hinter ihrem Verhalten als «Dickkopf», hinweisen.

Aus dieser Situation gleichsam herausgerissen, gerieten für Anita die ersten, zu häufig einsamen und sprachlosen Monate in der Schweiz zum traumatisierenden Schock. In unserer Alltagswahrnehmung, aber auch in der europäischen Migrationsforschung, werden psychische und soziale Konflikte von Migrantinnen und Migranten gerne mit dem Verlust von «Vaterland» und «Muttersprache» in Verbindung gebracht, das heisst, mit der Abwesenheit von Zugehörigkeit und Geborgenheit, die primär in einer väterlichen oder mütterlichen Metaphorik vorgestellt wird. Ich kann die beispielsweise von Yecheskiel Cohen (2005, S. 23) formulierte Analogie zwischen Migration und Geburt um eine weitere, für Anita mindestens ebenso wichtige Analogie ergänzen: Es handelt sich auf den kürzesten Nenner gebracht um die Frage nach der eigenen sozialen Position. Die Migrationssituation ist hier durch Faktoren charakterisiert, die weniger der Erfahrung der eigenen Geburt ähneln. Die Katastrophe, der Anita über längere Zeit ausgesetzt war, stellt eher eine Wiederholung des Moments der Deplatzierung durch die Geburt eines jüngeren Geschwisters dar. Die Migrationserfahrung hatte deshalb vor allem aus inneren Gründen traumatisierende Auswirkungen.

Dieses Szenario wird zudem untrennbar mit der Frage nach der geschlechtlichen Position, den geschlechtlichen Identifizierungen verknüpft sein, da auch das Geschlecht die Funktion eines sozialen Platzanweisers innehat. Anita hat nicht erst in ihrer Adoleszenz versucht, sich dieser Funktion zu widersetzen und - wie sich später zeigen wird - lenkte sie durch die Eheschliessung nur scheinbar ein. Anita suchte Abstand von ihrer Familie, sie suchte Sicherheit und Verlässlichkeit und fand diese auch in ihrer Partnerschaft. Die Gründung einer eigenen Familie mit Kindern hat sie aber im Grunde nie interessiert, worüber sie sich aber selbst nicht im Klaren war. Im wohl ziemlich durchschnittlichen schweizerischen Alltag ihrer Ehe, der im Unterschied zu indonesischen Verhältnissen mit langen Arbeitstagen des Ehemannes, dann wiederum vergleichsweise viel Nähe und Zweisamkeit in dessen Freizeit verbunden ist, findet sie schliesslich zu altbekannter Unzufriedenheit zurück und sucht nach Schuldigen und Rivalen. Wechselweise wendet sich ihre Wut nach aussen oder in der Form von depressiven Zuständen nach innen. Wie sehr Anitas Wortwahl bei Schilderungen von Konflikten mit ihrem Ehemann oder mit FreundInnen derjenigen beim Sprechen über ihre Geschwister ähnelt, beschreibe ich in meinem Buch. 
Anita war auf der Suche nach einem guten, einem dritten Ort, wo sie in ihren Fantasien von Null auf anfangen könnte, wo sie dem riesigen inneren Druck endlich entkommen könnte, wo man sie als das sehen würde, was sie ist. Ich habe sie gesehen und lieb gewonnen. In unseren Gesprächen hat Anita in Ruhe über einen dritten Ort fantasieren können. Unsere Gespräche endeten zu einem Zeitpunkt, wo Anita gerade begonnen hatte, es sich in ihrem gegenwärtigen Leben auf ihre Art und Weise etwas «bequemer» einzurichten.

\section{Der Geschwisterkomplex}

Vor der Ankunft oder dem «Eindringen», beziehungsweise «Kennenlernen» (Lacan, 1994, S. 54 ff.) von Geschwistern - bestehe dieses Ereignis nun in der realen Geburt eines Geschwisterkindes oder im reifebedingten Erwachen von Fantasien und Konzeptbildungen über ältere oder fehlende Geschwister - hat das Menschenwesen eine ihm gleichsam existenziell geschuldete Position inne. Ein Baby oder Kleinkind befinden sich bis zum Alter von ein bis zwei Jahren direkt am Körper oder meist in unmittelbarer Nähe der Mutter oder eines Mutterersatzes. Der mehr oder weniger plötzliche Verlust dieses Platzes wird je nach Kindesalter und individuellen Anlagen auf unterschiedliche Art und Weise, in der Regel jedoch als zutiefst beunruhigendes und verunsicherndes Ereignis erlebt. Nachträglich wird vom Subjekt ein imaginäres geschwisterloses «Paradies» konstruiert, dessen Verlust entweder betrauert und mehr oder weniger gut integriert oder aber verleugnet wird. Die Verleugnung kommt quasi einem anhaltenden unbewussten Anspruch auf dieses Paradies, das es so nie gegeben hat, gleich. In jedem Fall muss sich das von seinem Platz verdrängte Kind mit seiner neuen Position und mit der Tatsache befassen, dass es scheinbar irgendwie ersetzbar ist, dass es auch noch andere als es selbst gibt. Dann sucht das Kind den verlorenen Halt im erst jetzt sexualisierten ödipalen Dreieck. Der Kastrationskomplex konstituiert sich in dieser Sichtweise also in der Folge des Geschwisterkomplexes und beide seelisch strukturierenden Komplexe entfalten im Modus der Nachträglichkeit ihre Auswirkungen.

Diese andere Reihenfolge der Komplexe wird beim frühen Lacan entworfen: Es gibt die Vorstellung eines psychisch organisierenden Komplexes, der zwischen dem Komplex der Entwöhnung und dem Ödipuskomplex situiert ist. Lacan nannte ihn den «Komplex des Eindringlings» (Lacan, 1994). Die Tatsache und der Zeitpunkt seines Erscheinens bestimmen seine Bedeutung für das Subjekt.

Im vertikalen Paradigma von Freud wird hingegen dem Mutter-Vater-KindDreieck die primäre, im Grunde die alles entscheidende Funktion für die Ausbildung der psychischen Strukturen und somit des Subjekts zugeschrieben. Ähnliches gilt 
wohl für Lacan mit Ausnahme seiner letzten Schriften. Die psychischen Folgen des Geschwisterkomplexes werden durch eine vertikalisierende «Brille» hindurch gelesen. Die ersten Lebensjahre sind geprägt durch die existenzielle Abhängigkeit von versorgenden und auch emotional nährenden Eltern. Deshalb stehen alle weiteren Beziehungen psychisch in der Nachfolge dieser frühesten Abhängigkeit. In der abendländischen Geistesgeschichte und Psychologie erhielt diese frühkindliche Perspektive der absoluten Abhängigkeit einen paradigmatischen Status. Die psychische Konfrontation mit «Geschwistern» muss jedoch als Ausgangspunkt und somit strukturelle Weichenstellung für alle anderen lateralen sozialen Beziehungen eines Subjektes aufgefasst werden.

\section{4 Über-Ich, Gruppen-Ich, familiales Selbst oder communal conscience?}

Der Ethnopsychoanalytiker Paul Parin näherte sich in seinen theoretischen Schriften durchaus einer lateralen Sichtweise an. Auf der Grundlage seiner Westafrika-Forschungen stellt er beispielsweise fest, dass die Dogon-Gesellschaft in hierarchische Altersklassen gegliedert ist und dass diese Rangordnung «mehr Gewicht hat, als die ¿Zentralgewalt` des Ältesten oder der älteren Generation» (Parin, Morgenthaler \& Parin-Matthèy, 1989, S. 55). Eine laterale und nach Geschlechtern getrennte Hierarchie hat hier also im Alltag «mehr Gewicht» als die Rangordnung zwischen Eltern und Söhnen oder Töchtern. Die vertikale Gesellschaftsordnung der Dogon werde von einer «horizontalen» ergänzt, so Parin, der unter anderem ausgehend von dieser Beobachtung seine Konzeptualisierung eines «Gruppen-Ichs» entwickelte. Parin beschreibt, wie dieses «Gruppen-Ich» der Dogon auf bestimmte soziale Rahmenbedingungen angewiesen ist.

Sind in einer sozialen Situation Gemeinschaften oder Gruppen vorhanden, in denen wegen ihrer Struktur und der besonderen Psychologie der Mitglieder gegenseitige brüderliche bzw. schwesterliche Identifikationen möglich sind, ist das Gruppen-Ich ein Garant für eine gute soziale Anpassung (z. B. im Dogon Dorf). Dieser Anpassungsmechanismus gibt die Gemeinschaftsstruktur der Gesellschaft genauer wieder als andere. In der Kleinfamilie hat er keinen Platz. Im öffentlichen Leben der Industriegesellschaften muss er versagen. (Parin, 1978, S. 86-87)

Die Stringenz dieser der Absicht nach kulturvergleichenden Argumentation leidet indessen durch Parins Eingeständnis, man müsse durchaus auch Europäern 
ein Gruppen-Ich zuschreiben. So meint Parin, sie hätten vielleicht das Gruppen-Ich bei den Afrikanern leichter erkennen können, «weil uns ihre Abhängigkeit von der sozialen Umwelt deutlicher wurde als die von europäischen Analysanden, deren Abhängigkeiten den unseren gleichen» (Parin, 1978, S. 87).

Nicht nur, dass wir uns als Forscher in der eigenen Gesellschaft zuweilen im allzu ähnlichen Gegenüber spiegeln, ohne Differentes zu erkennen - dieselben Wahrnehmungsvoraussetzungen finden sich in der Theoriebildung und in der klinischen Praxis wieder und verstärken so die Tendenz, sich gedanklich um die eigene Achse zu drehen. Auch Parin, Parin-Matthèy und Morgenthaler ist es nicht gelungen, sich das ödipale und vertikalisierende Denkschema der Psychoanalyse genügend fremd oder andersherum, sich die lateralen Abhängigkeiten der fremden Analysanden genügend vertraut zu machen. Sie haben aber nach ihrer Rückkehr aus Afrika in der eigenen Gesellschaft ähnliche psychische Strukturen und Mechanismen wiedergefunden: Tatsächlich üben hinter der häufig brüchigen Fassade der Autonomie die lateral ausgerichteten Identifikationen und Leidenschaften auch bei den Subjekten in westeuropäischen Gesellschaften ihre eigentlich unverkennbare Wirkung aus. Diese gegenüber dem elterlichen Einfluss als sekundär, als weniger wichtig oder mächtig aufzufassen, erscheint mir theoretisch nicht haltbar. Parin hat jedoch aus seinen Erfahrungen meines Wissens nicht den Schluss gezogen, dass im Paradigma der psychoanalytischen TheoriebildungWesentliches fehlt. Was er für afrikanische Gesellschaften beschrieben hat, könnte aber auch heute noch zu diesen Lücken in der psychoanalytischen Theoriebildung hinführen.

Eine Auseinandersetzung mit kulturell unterschiedlichen psychischen Strukturierungen findet man selbstverständlich nicht nur in der deutschsprachigen Ethnopsychoanalyse, sondern allgemein in der psychoanalytisch orientierten Sozialanthropologie. Das Verhältnis von Universalismus zu Partikularismus wird unterschiedlich gewichtet. Vereinfacht gesagt geht ein Teil der AutorInnen stärker davon aus, dass alle Menschen sich in ihrer grundlegenden psychischen (Trieb-) Ausstattung ähnlich sind, andere wiederum betonen stärker die Differenz. So vertritt etwa Alan Roland (2005, S. 80) die Ansicht, der kulturelle Einfluss reiche bis in die tiefsten Schichten der Psyche. Er beschreibt verschiedene Dimensionen eines «familialen Selbst» bei Inderinnen und Indern, das stark beziehungs- und kontextorientiert funktioniere. Gananath Obeyesekeres Ausführungen hingegen gründen in einer anderen wissenschaftsphilosophischen Haltung. Er sucht über die Theoretisierung struktureller Differenz und empirischer Partikularität dennoch nach dem universell Menschlichen: 
Thus good ethnography is an ideal typical description that probes beneath the surface reality through the use of a nomological theory. Theory then takes us away from the particularities of X or Y culture and tells us something about culture or life in general. [...] Theory has an ontological thrust in the human sciences, since it is about human beings and being human that we are talking about. (Obeyesekere, 1990, S. 225)

Obeyesekere setzt sich mit Zusammenhängen zwischen Sozialstruktur und dem Ödipus-Komplex in unterschiedlichen südasiatischen Kontexten auseinander. Er stellt fest, dass die emotional wichtigen Beziehungen für ein Kind nicht nur aus denjenigen zu Mutter und Vater, sondern mindestens zusätzlich noch aus denjenigen zu Geschwistern bestehen. Laut Obeyesekere gibt es keinen universellen «Kern-Ödipuskomplex» mit Variationen, sondern eine begrenzte Anzahl von unterschiedlichen Ausprägungen dieses Komplexes. Obeyesekere bestreitet dabei die strukturelle Gleichheit dieser unterschiedlichen Ausprägungen. Er beschreibt in Übereinstimmung mit Roland (2005), Kakar (2012) und anderen, dass zumindest bis in die 1990er Jahre, die psychische Strukturierung in Indien (Südasien) mit einer Unterdrückung von Individualismus einherging.

The focus is on the welfare of the patrilineal joint family and the larger institutions of the society - caste and the political order-that together conspire to suppress individualism (if not individuality) in the interests of the collectivity. Kakar, following Erikson, has labelled these internalized norms as the "communal conscience". (Obeyesekere, 1990, S. 83)

Vorurteile finden sich auch im wissenschaftlichen Diskurs des sozialen Wandels, wo das «individualisierte» Leben in der Moderne auch heute noch mehr oder weniger unreflektiert einem gruppen- oder familienbezogenen, so genannt «traditionellen» Leben gegenübergestellt wird. Die Realität ist jedoch komplexer. Ich halte eine kulturelle Differenz-Setzung wie zum Beispiel diejenige zwischen «Über-Ich» und «Gruppen-Ich» für allzu grob und auch irreführend. Es gilt vielmehr, im Sinne Obeyesekeres, die unterschiedlichen Ausprägungen und Strukturierungen, die sich auf der Basis menschenmöglicher Konstellationen ausbilden, zu erforschen. 
In meiner Forschungsarbeit war ich vielfach damit konfrontiert, dass die eingewanderten Frauen den Verlust des alltäglichen Kontaktes mit nahestehenden Menschen sowie die Verunsicherung durch die neue(n) Sprache(n) und das Wegfallen von vertrauten Umgangsformen als anhaltendes Problem erlebten. Im neuen Lebenskontext brauchen Migrantinnen und Migranten neue Freunde und Ersatz-Verwandte. Ob und wie sie dabei auf Geschwisterersatz oder stärker auf Elternfiguren ausgerichtet sind, ob sie eher nach unverbindlichen oder nach engen, von gegenseitigen Verpflichtungen charakterisierten Beziehungen suchen oder nach beidem, auf welche Weise sie dabei erfolgreich sind oder Schwierigkeiten erleben, das bleibt im Einzelfall zu analysieren. Ich habe zu differenzieren versucht, unter welchen Umständen welche Schwierigkeiten zu unüberwindlichen Hindernissen werden und wann diese zwar stören oder Leiden verursachen, einen psychosozialen Integrationsprozess aber nicht verunmöglichen. Ich sah mich dabei vor die Aufgabe gestellt, bei jedem Einzelfall jeweils äussere strukturelle Integrationsbarrieren von inneren strukturellen Integrationshemmnissen und von fördernden Faktoren zu unterscheiden und zu versuchen deren dialektisches Verhältnis zu verstehen.

Das Funktionieren einer Einwanderungsgesellschaft hängt von den Möglichkeiten ab, welche die zunächst «Fremden» für ein Wieder-Erschaffen von Beziehungsstrukturen haben. Und die Einwanderungsgesellschaft wiederum ist abhängig von ihren «Fremden» oder «Eindringlingen».

\section{Das Migrations-Trauma und der Geschwisterkomplex}

Wir glauben, dass die spezifische Qualität der Reaktion aufdie traumatische Erfahrung der Migration das Gefühl der "Verlassenheit» ist. Dieses Gefühl der Verlassenheit basiert ursprünglich auf dem Geburtstrauma (Rank, 1924) und dem Verlust der schützenden Mutter. [...] Das Risiko ist grösser, wenn im Laufe der Kindheit wichtige Entbehrungs- und Trennungssituationen und darauf folgende Angst- und Verlassenheitserfahrungen erlitten wurden. (Grinberg \& Grinberg, 1990, S. 12)

In diesem Klassiker der psychoanalytischen Migrationsliteratur benennt das Autorenpaar Léon und Rebeca Grinberg das Gefühl der «Verlassenheit» als die besondere Qualität «der Reaktion auf die traumatische Erfahrung der Migration». Die Autoren führen anhand von Fallbeispielen vor, wie sich diese Gefühlsqualität 
von Verlassenheit im Einzelfall unterschiedlich zeigt und entwickelt. Anders als Mitchell betonen sie theoretisch den Beginn der extrauterinen menschlichen Existenz anhand des von Otto Rank entwickelten Konzepts des «Geburtstraumas». Mit dem Herstellen einer Analogie zwischen der Situation des Neugeborenen und derjenigen des Migranten schliessen sie logisch an dieses Konzept an. Wie ich bereits weiter vorne angesprochen habe, wird das Herkunftsland so zum verlorenen «Mutterland», was zur verbreiteten Idealisierung der verlassenen Heimat oder Herkunftskultur durch Auswanderer passt. Aus dieser vertikalen Mutter-KindPerspektive werden zweifelsohne wichtige Zusammenhänge beschreibbar. Auf der Grundlage einer entsprechenden Metaphorik erläutern die Grinbergs auch die Möglichkeit, die Krise der Migration schliesslich als bereichernde «WiederGeburt» zu erleben.

Zusätzlich zu diesem Gefühl von Verlassenheit haben sich in meinen Fallanalysen andere Erfahrungsqualitäten herauskristallisiert. Ich benenne diese hier noch einmal: Gerade noch war ich jemand, jetzt bin ich plötzlich niemand mehr. Oder auch: Welcher Platz ist für mich vorgesehen? Mir erscheinen alle wichtigen Positionen bereits besetzt. Ich versuche mich gleich zu verhalten, wie meine neuen Geschwister, aber diese reagieren auf mich nicht, wie ich es erwartet habe. Sind mir die anderen überhaupt wohlgesinnt oder stehen sie mir ablehnend, feindlich gegenüber? Nicht Verlassenheit wäre die spezifische dazugehörige Gefühlsqualität, sondern eher Gefühle von vernichtender Zurücksetzung, von paranoid gefärbter Angst vor feindseliger Verfolgung bis hin zu geraubter oder nicht zugebilligter Existenzberechtigung.

Es versteht sich von selbst, dass auch die jeweiligen politischen und sozialen Umstände einer Migration für deren Erleben mitentscheidend sind. Entsprechend unterschiedlich sehen die Integrationsprozesse bei Flüchtlingen und bei freiwillig Migrierenden, bei jungen und älteren Menschen, bei Gesunden und Kranken, bei so genannt «gerufenen» oder «ungebetenen», bei deutschen, portugiesischen, afrikanischen oder indonesischen, bei mausarmen oder vermögenden Zugewanderten aus. Aber alle können sie sich in der Gesellschaft des Einwanderungslandes mehr oder weniger deplatziert fühlen. In denWorten von Mitchell lautet die dazugehörige Frage, die sich dem Subjekt stellt: "The struggle is not, Who am I? but, Where am I?; not one of identity (though it is often confused with this) but of, What is my position in this kinship scenario?" (Mitchell, 2000, S. 329).

Diese Erfahrung mag in den meisten Fällen verhältnismässig unkompliziert und erfolgreich verlaufen; sie kann aber ebenso - zuweilen um Jahre verzögert - in eine schwerere psychische Krise münden und sich als Traumatisierung 
auswirken. Geburtstrauma, Entwöhnung, Geschwisterkomplex, Ödipus- oder Kastrationskomplex, so lauten die wichtigsten strukturellen Entwicklungsaufgaben und Gefährdungen aus psychoanalytischer Sicht. Wenn sich die frühe Katastrophe des Realisierens der Ersetzbarkeit oder «Verrückbarkeit» bei einem Kind, aus welchen Gründen auch immer, allzu tief eingräbt, wenn es nicht in der Lage ist, gewisse Identifizierungen aufzugeben oder gleichsam zu relativieren, dann kann laut Mitchell ein gegenwärtig unangenehmes oder überforderndes Ereignis unbewusst als Wiederholung der früheren Katastrophe erlebt und mit entsprechenden psychischen Folgen als Trauma agiert werden.

Auch bei relativ günstigen und freundlichen äusseren Bedingungen kann eine Migrantin scheitern, die durch eine oder mehrere dieser allgemein menschlichen Entwicklungsaufgaben bereits in der Kindheit überfordert war, was eine besondere psychische Fragilität zur Folge hat. Um eine derartige Krise zu überwinden oder erst gar nicht in sie hineinzugeraten, ist es für Migrantinnen und Migranten notwendig, sich einen «Übergangsraum» zu schaffen, gleichsam einen dritten, hybriden oder «richtigen» Ort, der irgendwo zwischen Herkunfts- und Ankunftsland, zwischen Innen und Aussen sowie zwischen den Zeiten angesiedelt ist und der zunächst spielerisch neu belebt werden kann. Die meisten AutorInnen, die von einem solchen «Übergangsraum» sprechen, beziehen sich auf Konzepte des englischen Psychoanalytikers Donald Winnicott. Der Begriff des «Übergangsraumes» wird von Maya Nadig für die ethnopsychoanalytische Forschungssituation verwendet (vgl. Nadig, 2000; 2006; vgl. auch zur «Therapie als Übergangsraum» bei Saller, 2003, S. 312 ff.; zum Begriff des «interkulturellen Entwicklungsraumes» bei Utari-Witt, 2005; zum Begriff des «transkulturellen Übergangsraums» bei Özbek et al., 2006). Idealerweise kann in diesem Übergangsraum das Wagnis eingegangen werden, sowohl die vertikale wie auch die laterale Achse des «Verwandtschaftsszenarios» neu aufzubauen. Dieses soziale Szenario muss unter Umständen stark veränderten Bedingungen genügen. Und es ist ein notwendiges Erfordernis, die Merkmale der eigenen sozialen Position darin neu zu symbolisieren.

Neben der Partikularität und der Kontingenz von Einzelschicksalen geht es bei Geschwistern zugleich um die weitreichende Frage nach der Geschlechterdifferenz und der sozialen Reproduktion von Gesellschaften. Der Topos der inzestuösen Liebe zwischen Geschwistern, beziehungsweise des Inzesttabus/Exogamiegebotes gehört gleichzeitig zu den begründenden Mythen sowohl der Ethnologie als auch der Psychoanalyse. Dieser Topos ist im Zuge des gesellschaftlichen Wandels bereits seit einiger Zeit von Verschiebungen oder, wie manche meinen, von Korrosion betroffen. Der Geschlechterdifferenz und dem Inzestverbot stehen heute das Gebot 
der Anerkennung pluraler Differenzen und Identitäten sowie das Recht auf körperliche und sexuelle Selbstbestimmung gegenüber. Als Anzeichen dieser zumindest von einer Art Verschiebung betroffenen symbolischen Grenzziehungen, kann der umstrittene Vorschlag der Aufhebung des Straftatbestandes des Inzests zwischen erwachsenen Verwandten und Geschwistern gelesen werden, der $2010 \mathrm{im}$ Zuge der letzten Revision des Schweizerischen Strafgesetzes vom Bundesrat vorgebracht wurde. In meiner Dissertation (2009) habe ich der Frage der Wandelbarkeit des sozialen und psychischen Geschlechts, die ich hier nur streifen kann, ein eigenes Kapitel gewidmet.

Geschwister- und Peer-Bindungen sind als früheste soziale Beziehungen prägend. Es geht bei den Geschwistern um diejenigen, die uns in vielem ähnlicher als sonst irgendjemand und die doch so anders sind, um diejenigen also, in denen wir uns spiegeln und wiedererkennen, die uns gleichzeitig besonders nahestehen oder aber fremder als sonst irgendjemand sein können. Selbstliebe, Liebe und Hass - at its best and at its worst - gehören zu diesem lateralen Beziehungskomplex. Wir erfahren durch Geschwister, wo unser Platz in der Welt ist und wo er sein könnte und unser Begehren strukturiert sich wesentlich entlang dieser lateralen Achse.

\section{Literatur}

Bally, A. (2009). Fremde Schwestern. Aus den Lebensgeschichten indonesischer Frauen in der Schweiz: Ethnologische und psychoanalytische Analysen unter besonderer Berücksichtigung der Geschwisterdimension. Unveröffentlichte Inauguraldissertation: Universität Bern.

Bally, A. (2013). Die Macht der Geschwister. Indonesische Frauen in der Schweiz: Eine ethnologische und psychoanalytische Migrationsforschung. Frankfurt a. M.: Campus.

Binderhofer, E. (2003). Der Himmel in meinem Land hat eine andere Farbe. LebensGeschichten - Gespräche mit asiatischen Frauen in Wien. Wien: Edition Roesner.

Cohen, Y. (2005). Frühe Entwicklung und Migrationsprozesse. In P. Bründl et al. (Hrsg.), Kindheit jenseits von Trauma und Fremdheit. Psychoanalytische Erkundungen von Migrationsschicksalen im Kindes- und Jugendalter (S. 17-29). Frankfurt a. M.: Brandes \& Apsel.

Feller, A. \& Zemp, J. (2006). Renah Kemumu - Ein indonesisches Dorf im Wandel der Zeit. Heiratsstrategien und Migrationsverhalten im Spiegel einer Verwandtschaftsgenealogie. Masterarbeit, Universität Bern. 
Freud, S. (1999). Analyse der Phobie eines fünfjährigen Knaben. GW, Bd. VII. Frankfurt a. M.: Fischer. [Original erschienen 1909].

Grinberg, L. \& Grinberg, R. (1990). Psychoanalyse der Migration und des Exils. München: Verlag Internationale Psychoanalyse.

Kakar, S. (2012). Die Seele der Anderen. Mein Leben zwischen Indien und dem Westen. München: C.H. Beck Verlag.

Lacan, J. (1994). Schriften III. Weinheim, Berlin: Quadriga. [Original erschienen 1966, Paris: Editions du Seuil].

Marschall, W. (2000). Brüder und Schwestern. Einige ethnologische Bemerkungen zu Geschwistern. In G. Klosinski (Hrsg.), Verschwistert mit Leib und Seele (S. 31-40). Attempto: Tübingen.

McKinley, R. (1983). Cain and Abel on the Malay Peninsula. In M. Marshall (Hrsg.), Siblingship in Oceania-Studies in the Meaning of Kin Relations (S. 335-387). Boston: University Press of America.

Mitchell, J. (2000). Mad Men and Medusas. Reclaiming Hysteria. New York: Basic Books.

Mitchell, J. (2003). Siblings. Sex and Violence. Cambridge: Polity Press.

Nadig, M. (2000). Interkulturalität im Prozess. Ethnopsychoanalyse und Feldforschung als methodischer und theoretischer Übergangsraum. In H. Lahme-Gronostay \& M. Leuzinger-Bohleber (Hrsg.), Identität und Differenz. Zur Psychoanalyse des Geschlechterverhältnisses in der Spätmoderne (S. 87-101). Wiesbaden: Westdeutscher Verlag.

Nadig, M. (2006). Transkulturelle Spannungsfelder in der Migration und ihre Erforschung. In E. Wohlfahrt \& M. Zaumseil (Hrsg.), Transkulturelle Psychiatrie - Interkulturelle Psychotherapie. Interdisziplinäre Theorie und Praxis (S. 67-80). Heidelberg: Springer Verlag.

Obeyesekere, G. (1990). The Work of Culture. Symbolic Transformation in Psychoanalysis and Anthropology. Chicago: The University of Chicago Press.

Özbek, T. \& Wohlfahrt, E. (2006). Der transkulturelle Übergangsraum - ein Theorem und seine Funktion in der transkulturellen Psychotherapie am ZIPP. In E. Wohlfahrt \& M. Zaumseil (Hrsg.), Transkulturelle Psychiatrie Interkulturelle Psychotherapie (S. 169-176). Heidelberg: Springer Verlag.

Parin, P. (1978). Der Widerspruch im Subjekt. Ethnopsychoanalytische Studien. Frankfurt a. M.: Syndikat. 
Parin, P., Morgenthaler, F. \& Parin-Matthèy, G. (1989). Die Weissen denken zuviel. Psychoanalytische Untersuchungen bei den Dogon in Westafrika. Frankfurt a. M.: Fischer.

Peletz, M. (1988). A Share of the Harvest: Kinship, Property, and Social History among the Malays of Rembau. Berkeley: University of California Press.

Roland, A. (2005). Multiple Mothering and the Familial Self. In S. Akhtar (Hrsg.), Freud along the Ganges. Psychoanalytic Reflections on the People and Culture of India (S. 79-90). New York: Other Press.

Saller, V. (2003). Wanderungen zwischen Ethnologie und Psychoanalyse. Psychoanalytische Gespräche mit Migrantinnen aus der Türkei. Tübingen: edition diskord.

Schneider, J. (1993). Kakak und Adik statt Brüder und Schwestern: Geschwisterbeziehungen in Indonesien. rebus, 3, 41-50.

Smith Kipp, R. (1986). Terms of Endearment: Karo Batak Lovers as Siblings. American Ethnologist, 13(4), 632-645.

Utari-Witt, H. (2005). Begegnung im interkulturellen Entwicklungsraum. In P. Bründl, et al. (Hrsg.). Kindheit jenseits von Trauma und Fremdheit. Psychoanalytische Erkundungen von Migrationsschicksalen im Kindes- und Jugendalter (S. 190-216). Frankfurt a. M.: Brandes \& Apsel. 